\title{
Local Realism vs Quantum Mechanics with Entangled Neutral Kaons
}

\author{
Albert Bramon ${ }^{a}$ and Gianni Garbarino ${ }^{* b}$ \\ ${ }^{a}$ Grup de Física Teòrica, Universitat Autònoma de Barcelona, E-08193 Bellaterra, Spain \\ ${ }^{b}$ Dipartimento di Fisica Teorica, Università di Torino and INFN, Sezione di Torino, I-10125 \\ Torino, Italy \\ E-mail: bramoneifae.es garbarindto.infn.it
}

We shortly review a couple of proposals of Bell inequality tests with entangled pairs of neutral kaons from $\phi$ resonance decays. The suggested experiments are discussed in the light of the essential requirements for a genuine discrimination between local hidden-variable theories (i.e., local realism) and quantum mechanics. We clarify the logical limitations of these tests in refuting the whole class of local realistic models. Detailed analyses imply that a Bell-type test with neutral kaons free from the detection loophole requires a few $\%$ strangeness detection efficiencies and very high efficiencies for the detection of the kaon decay products.

PACS : $03.65 .-\mathrm{w}, 03.65 . \mathrm{Ud}, 14.40 . \mathrm{Aq}$

Kaon International Conference

May 21-25, 2007

Laboratori Nazionali di Frascati dell'INFN

\footnotetext{
*Speaker.
} 
1. Introduction - The correlations shown by the distant parts of a system in an entangled state offer one of the most counterintuitive and subtle aspects of quantum mechanics. This became evident with the appearance of the well known papers by Einstein-Podolsky-Rosen (EPR) and Bohr [1] on the completeness of quantum mechanics. For about 30 years the debate triggered by EPR and Bohr was reputed to be a philosophical matter rather than an actual physical question. In 1964, Bell [2] interpreted EPR's argument as the need for the introduction of additional, unobservable variables aiming to restore completeness, relativistic causality (i.e., locality) and realism in quantum theory. He established a theorem which proved that any local hidden-variable (i. e., local realistic) theory is incompatible with some statistical predictions of quantum mechanics. Since then, various forms of Bell inequalities [3]-[ [5] have been the usual tool for an experimental discrimination between local realism (LR) and quantum mechanics (QM).

Bell-type tests have been performed mainly with entangled photons [5, [6] and ions [7]. Although these tests obtained results in good agreement with QM, they do not represent a conclusive proof against LR since they only showed the violation of the so called non-genuine Bell inequalities. Because of non-idealities of the apparata and other technical problems, supplementary assumptions not implicit in LR were needed in the interpretation of these experiments. No one of these tests has been loophole-free $\mathrm{G}, \mathrm{B}$, i. e., able to test a genuine Bell inequality, which is a consequence of LR alone. Two main logical loopholes affected the performed experiments. The detection loophole originates from the fact that the achievable detection effi ciencies are lower than the thresholds required for the violation of genuine inequalities 15. 9]. In addition, an experiment is not free from the locality loophole if the measurements on the two parties of the entangled state are not carried out under strict space-like separation conditions; exchange of subluminal signals between the two measurement event regions cannot be avoided in such cases.

Extensions to other kinds of entangled systems are thus important. Recently, there has been an increased interest on the possibility to test LR in particle physics, by using entangled neutral kaons [10]-[15]. In $\phi$ resonance decays, as at DaФne [16], or $p \bar{p}$ annihilation processes at rest [17], neutral kaon pairs are produced in the maximally entangled state:

$$
|\phi(0)\rangle=\left\{\left|K^{0}\right\rangle_{l}\left|\bar{K}^{0}\right\rangle_{r}-\left|\bar{K}^{0}\right\rangle_{l}\left|K^{0}\right\rangle_{r}\right\} / \sqrt{2}=\left\{\left|K_{S}\right\rangle_{l}\left|K_{L}\right\rangle_{r}-\left|K_{L}\right\rangle_{l}\left|K_{S}\right\rangle_{r}\right\} / \sqrt{2}
$$

where $l$ and $r$ denote the 'left' and 'right' kaon directions of motions and $C P$ violation effects have been neglected in the second equality. The strong nature of kaon hadronic interactions has the value of enhancing the effi ciencies to detect the products of kaon decays and interactions with ordinary matter. Moreover, the measurements on each one of the two kaons produced in $\phi$ decays or $p \bar{p}$ annihilations at rest can be performed when they are separated by many centimeters. Closing the locality loophole is then a hard, but merely technical, problem. However, other specifi c diffi culties appear with kaons which renders very diffi cult a genuine test of LR. In this contribution we concentrate on these diffi culties and discuss a couple of kaonic Bell-type tests in the light of the basic requirements necessary to establish genuine tests of LR. For detailed reviews on the subject see Refs. [13, 14, 15.

2. Requirements for a genuine Bell inequality test - The requirements for deriving a genuine Bell inequality from LR can be summarized as follows: (1) A non-factorisable or entangled state must be used; (2) Alternative and mutually exclusive measurements, corresponding to non-commuting observables, must be chosen at will and performed on both members of that state (reality requirement); (3) Each one of the different single measurements has to have dichotomic outcomes; (4) The measurement process on each member of the two-particle state must be space-like separated from the measurement on the other member (locality requirement).

At a $\phi$-factory, or in $p \bar{p}$ annihilations at rest, the fi rst requirement poses no serious problems: entanglement has been confi rmed experimentally for $K^{0} \bar{K}^{0}$ pairs $[17,18]$. Diffi culties appear with requirements number (2) and (4) and are discussed in the next Section. In order to establish the feasibility of the real test, one fi nally has to derive the minimum detection effi ciencies necessary for a meaningful QM violation of the considered Bell inequality.

3. Neutral kaon measurement procedures - According to previous requirements (2) and (4), for genuine Bell-type tests the adopted measurements must be performed by exerting the free will of the experimenters in two space-like separated regions. Measurements on neutral kaons reduce to only two kinds. At the chosen measurement time, one can either place a slab of ordinary matter or allow for kaon free-space propagation, thus measuring either strangeness or lifetime. Actively changing from one setup to another in such a way that the two measurement events are space-like separated implies serious technical diffi culties. The use of fully passive measurements, which are also possible for kaons and are important for other QM tests [19], prevents the derivation of genuine Bell inequalities [20].

Strangeness - The 'strangeness' basis consists of the two eigenstates $\left|K^{0}\right\rangle$ and $\left|\bar{K}^{0}\right\rangle$ with strangeness $S=+1$ and $S=-1$, respectively, and is suitable to analyze $S$-conserving electromagnetic and strong interaction processes. 
Strangeness of a given kaon state is measured by placing a piece of ordinary material along the meson path. When a kaon-nucleon reaction occurs, the distinct strong interactions of the $S=+1$ and $S=-1$ neutral kaons on the bound nucleons inside the medium project the arbitrary state of an incoming kaon into one of the two orthogonal members of the strangeness basis [13]. Unfortunately, the effi ciency for such strangeness measurements at moderate kaon energies is less than what people have been naively expecting from the strong nature of these interactions [17]. The reason, rather than being the diffi culty in detecting the fi nal state particles, stems from the low probability in initiating the strong reaction. Ordinary matter is indeed too transparent for kaons.

Lifetime - The short- and long-lived states, $\left|K_{S}\right\rangle$ and $\left|K_{L}\right\rangle$, are the normalized eigenvectors governing neutral kaon (proper) time evolution in free-space:

$$
\left|K_{S(L)}(\tau)\right\rangle=e^{-i m_{S, L} \tau} e^{-\frac{1}{2} \Gamma_{S(L)} \tau}\left|K_{S(L)}(\tau=0)\right\rangle \equiv e^{-i \lambda_{S(L)} \tau}\left|K_{S(L)}\right\rangle,
$$

$m_{S, L}$ and $\Gamma_{S, L}$ being the $K_{S, L}$ masses and decay widths. They defi ne the quasi-orthonormal lifetime basis, with $\left\langle K_{S} \mid K_{L}\right\rangle=$ $\left(\varepsilon+\varepsilon^{*}\right) /\left(1+|\varepsilon|^{2}\right), \varepsilon$ being a $C P$ violating parameter.

To measure if a kaon is propagating in free-space as a $K_{S}$ or $K_{L}$ at a given time $\tau$, one has to allow for further propagation in free-space and then detect at which time the kaon decays. Kaons decaying between times $\tau$ and $\tau+\Delta \tau$ have to be identifi ed as $K_{S}$ 's, while those decaying later than $\tau+\Delta \tau$ have to be identifi ed as $K_{L}$ 's. By choosing $\Delta \tau=4.8 \tau_{S}$, both $K_{S}$ and $K_{L}$ misidentifi cation probabilities reduce to $\simeq 0.8 \%$, which can be further decreased if the decay mode is also identifi ed (see appendix of Ref. [1] ]). At DaФne one has at disposal rather high detection effi ciency for these lifetime measurements.

\section{Kaonic Bell-type tests}

A proposal assuming fair sampling - We start by analyzing a proposal [11] based on the fair sampling hypothesis [5], which amounts to assume that the set of effectively measured events represent an undistorted sample of the whole set of states emitted from the source. While such an assumption represents an obvious procedure in quantum measurement, in a local realistic model the hidden-variables can be well correlated with the effi ciencies of measuring apparata. Under fair sampling, the effi ciency factors in the Bell inequalities are assumed to be 1 and thus no undetected event appears.

The entangled state used in Ref. [11] is prepared by placing a thin regenerator with parameter $\eta$ on the right beam, close to the point where the two-kaon state (1) originates. We assume that the proper time $\Delta \tau$ required by the right moving neutral kaon to cross the regenerator is short enough $\left(\Delta \tau<<\tau_{S}\right)$ to ignore kaon decays. Free propagation is then considered for the left and right beams up to a proper time $T$, with $\tau_{S}<<T<<\tau_{L} \simeq 579 \tau_{S}$. After normalization to surviving pairs, one obtains the non-maximally entangled state:

$$
|\Phi\rangle=\left\{\left|K_{S}\right\rangle\left|K_{L}\right\rangle-\left|K_{L}\right\rangle\left|K_{S}\right\rangle+R\left|K_{L}\right\rangle\left|K_{L}\right\rangle\right\} / \sqrt{2+|R|^{2}}, \quad R \equiv-\eta e^{\left[i\left(m_{S}-m_{L}\right)+\frac{1}{2}\left(\Gamma_{S}-\Gamma_{L}\right)\right] T} .
$$

For each kaon on each beam at time $T$, one then considers either a strangeness or a lifetime measurement. With the strategy adopted for lifetime measurements, requiring an extra interval time $\Delta T$ after $T$, for kaon pairs from $\phi$ decays, one has to use $T>1.77 \Delta T$ to guarantee the space-like separation between left and right measurements.

In Ref. [1] the following Clauser-Horne $(\mathrm{CH})$ inequalities [4] have been derived under the assumption of perfectly effi cient measuring apparata:

$$
\frac{P\left(\bar{K}^{0}, K_{L}\right)-P\left(\overline{K^{0}}, \bar{K}^{0}\right)+P\left(K_{S}, \bar{K}^{0}\right)+P\left(K_{S}, K_{L}\right)}{P\left(K_{S}, *\right)+P\left(*, K_{L}\right)} \leq 1, \quad \frac{P\left(\bar{K}^{0}, K_{S}\right)-P\left(\bar{K}^{0}, \bar{K}^{0}\right)+P\left(K_{L}, \bar{K}^{0}\right)+P\left(K_{L}, K_{S}\right)}{P\left(*, K_{S}\right)-P\left(K_{L}, *\right)} \leq 1,
$$

where, for instance, $P\left(K_{S}, *\right) \equiv P\left(K_{S}, K^{0}\right)+P\left(K_{S}, \bar{K}^{0}\right)$. These $\mathrm{CH}$ inequalities are satisfi ed by all local realistic models supplemented by fair sampling. By substituting the QM predictions, these inequalities imply: $\left(2-\mathscr{R} e R+|R|^{2} / 4\right) /(2+$ $\left.|R|^{2}\right) \leq 1,\left(2+\mathscr{R} e R+|R|^{2} / 4\right) /\left(2+|R|^{2}\right) \leq 1$. According to the sign of $\mathscr{R} e R$, one of these two inequalities is violated if $|\mathscr{R} e R| \geq 3|R|^{2} / 4$. The greatest violation occurs for a purely real value of $R,|R| \simeq 0.56$, for which one of the two previous ratios reaches the value 1.14. This $14 \%$ violating effect predicted by QM opens up the possibility for a meaningful kaonic Bell-type test which could refute those LR models incorporating fair sampling.

An attempt of genuine test - We now discuss a proposal which does not assume hypotheses beyond LR. In our opinion, it represent an interesting attempt for a loophole-free test of LR vs QM with neutral kaons.

Hardy's proof without inequalities of Bell theorem [21] has been applied in Ref. [12] to the non-maximally entangled state (3). Neglecting $C P$-violation and $K_{L}-K_{S}$ misidentifi cation effects, from state $\$$ ) with $R=-1$ (Hardy's state) one obtains the following $\mathrm{QM}$ predictions:

$$
P_{\mathrm{QM}}\left(K^{0}, \bar{K}^{0}\right)=\eta \bar{\eta} / 12, \quad P_{\mathrm{QM}}\left(K^{0}, K_{L}\right)=0, \quad P_{\mathrm{QM}}\left(K_{L}, \bar{K}^{0}\right)=0, \quad P_{\mathrm{QM}}\left(K_{S}, K_{S}\right)=0,
$$


where $\eta(\bar{\eta})$ is the overall effi ciency for $K^{0}\left(\bar{K}^{0}\right)$ detection. It is found that the necessity to reproduce, under LR, the fi rst three equalities in Eq. $\$$ ), requires $R_{\mathrm{LR}}\left(K_{S}, K_{S}\right) \geq P_{\mathrm{LR}}\left(K^{0}, \bar{K}^{0}\right)=\eta \bar{\eta} / 12>0$, which contradicts the last equality of Eq. (5). In principle, this allows for an 'all-or-nothing' test of LR vs QM. By requiring a perfect discrimination between $K_{S}$ and $K_{L}$ states, an experiment measuring the joint probabilities of Eq. ( $\$$ ) closes the effi ciency loophole even for infi nitesimal values of the strangeness detection effi ciencies [1 1 ]. However, since $K_{\mathbb{L}}$ and $K_{S}$ misidentifi cations do not permit an ideal lifetime measurement even when the detection effi ciency $\eta_{\tau}$ for the kaon decay products is 1 , the question must be reconsidered by paying attention to the ineffi ciencies involved in the real test.

Retaining the lifetime misidentifi cation effects, the predictions $[$ ) are replaced by (see Ref. [1] ] for details):

$$
\begin{aligned}
& P_{\mathrm{QM}}\left(K^{0}, \bar{K}^{0}\right)=\eta \bar{\eta} / 12, \\
& P_{\mathrm{QM}}\left(K^{0}, K_{L}\right)=6.77 \times 10^{-4} \eta \eta_{\tau}, \\
& P_{\mathrm{QM}}\left(K_{L}, \bar{K}^{0}\right)=6.77 \times 10^{-4} \bar{\eta} \eta_{\tau}, \\
& P_{\mathrm{QM}}\left(K_{S}, K_{S}\right)=1.19 \times 10^{-5} \eta_{\tau}^{2} .
\end{aligned}
$$

In the standard Hardy's proof of non-locality [21], the probabilities corresponding to our (1)-(6) are perfectly vanishing. In our case they are very small but not zero. Nevertheless, this does not prevent us from deriving a contradiction between LR and QM. Indeed, it turns out [15] that the following Eberhard inequality [9]

$$
H \equiv \frac{P\left(K^{0}, \bar{K}^{0}\right)}{P\left(K^{0}, K_{L}\right)+P\left(K_{S}, K_{S}\right)+P\left(K_{L}, \bar{K}^{0}\right)+P\left(K^{0}, U_{\text {Lif }}\right)+P\left(U_{\text {Lif }}, \bar{K}^{0}\right)} \leq 1 .
$$

must be used to demonstrate the incompatibility between LR and QM in the realistic case. The argument $U_{\text {Lif }}$ refers to failures in lifetime detection and $P_{\mathrm{QM}}\left(K^{0}, U_{\mathrm{Lif}}\right)=\eta\left(1-\eta_{\tau}\right) / 6, P_{\mathrm{QM}}\left(U_{\mathrm{Lif}}, \bar{K}^{0}\right)=\bar{\eta}\left(1-\eta_{\tau}\right) / 6$. It is important to stress that the previous Eberhard inequality has been obtained without invoking supplementary assumptions on undetected events, i.e., it is a genuine Bell inequality.

We now have to discuss the feasibility of the corresponding experiment. In the ideal case with no undetected events ( $\eta=\bar{\eta}=\eta_{\tau}=1$ ), the inequality is strongly violated by $\mathrm{QM}, H_{\mathrm{OM}}^{\eta=\bar{\eta}=\eta_{\tau}=1} \simeq 60.0$, even if one allows for unavoidable $K_{S}$ and $K_{L}$ misidentifi cations. Assuming that only the detection effi ciency of kaon decay products is ideal $(\eta=1)$, for $\eta=\bar{\eta}(\eta=\bar{\eta} / 2)$ Eberhard inequality is contradicted by QM whenever $\eta>0.023(\eta>0.017)$. Let us now consider more realistic situations with small and possibly achievable values of $\eta$ and $\bar{\eta}$. This implies that we have to consider large decay-product detection effi ciencies. For each $\eta_{t}$, the values of $\eta$ and $\bar{\eta}$ that permit a detection-loophole-free test $\left(H_{\mathrm{QM}}>1\right)$ lie above the corresponding curve plotted in Fig. 11. As expected, when $\eta_{\tau}$ decreases, the region of $\eta$ and $\bar{\eta}$ values which permits a conclusive test diminishes and larger values of $\eta$ and $\bar{\eta}$ are required.

In conclusion, a Bell-type test with neutral kaons and with strangeness detectors having an effi ciency of a few $\%$ would be free from the detection loophole if almost ideal lifetime measurements were achievable. In any case, as for the performed photon experiments, the proposed kaonic test is able to refute LR modulo the fair sampling assumption.

\section{References}

[1] A. Einstein, B. Podolsky and N. Rosen, Phys. Rev. 47, 777 (1935); N. Bohr, Phys. Rev. 48, 696 (1935).

[2] J. Bell, Physics 1, 195 (1964).

[3] J. F. Clauser, M. A. Horne, A. Shimony and R. A. Holt, Phys. Rev. Lett. 23, 880 (1969).

[4] J. F. Clauser and M. A. Horne, Phys. Rev. D 10, 526 (1974).

[5] J. F. Clauser and A. Shimony, Rep. Prog. Phys. 41, 1881 (1978).

[6] A. Aspect, J. Dalibard and G. Roger, Phys. Rev. Lett. 49, 1804 (1982); G. Weihs, T. Jennewein, C. Simon, H. Weinfurter and A. Zeilinger, Phys. Rev. Lett. 81, 5039 (1998); W. Tittel, J. Brendel, H. Zbinden and N. Gisin, Phys. Rev. Lett. 81, 3563 (1998).

[7] M. A. Rowe et al., Nature 409, 791 (2001).

[8] E. Santos, Phys. Lett. A 327, 33 (2004); Found. Phys. 34, 1643 (2004).

[9] P. H. Eberhard, Phys. Rev. A 47, R747 (1993). 


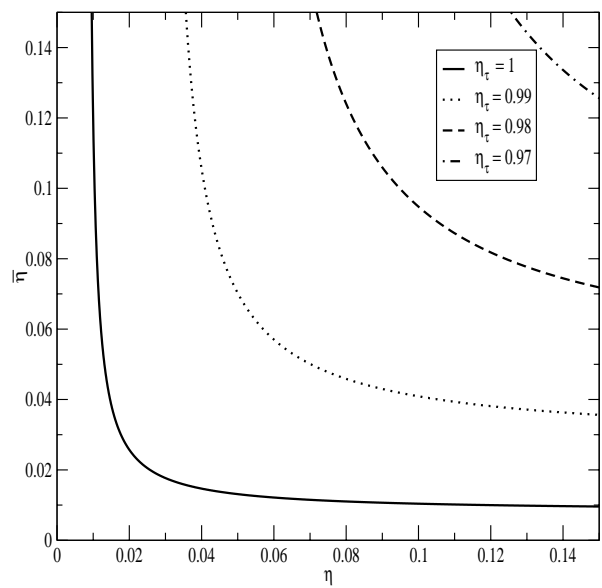

Figure 1: The four curves (corresponding to $\eta_{\tau}=1, \eta_{\tau}=0.99, \eta_{\tau}=0.98$ and $\eta_{\tau}=0.97$ ) provide the values of $\eta$ and $\bar{\eta}$ for which $H_{\mathrm{QM}}=1$. QM violates inequality (10) for values of $\eta$ and $\bar{\eta}$ situated above the corresponding curve.

[10] G. C. Ghirardi, R. Grassi and T. Webern, in Proceedings of the Workshop on Physics and Detectors for Da $\Phi$ ne, edited by G. Pancheri, p. 261 (INFN, LNF, Frascati, Italy, 1991); P. H. Eberhard, Nucl. Phys. B 398, 155 (1993); A. Di Domenico, Nucl. Phys. B 450, 293 (1995); F. Uchiyama, Phys. Lett. A 231, 295 (1997); R. Foadi and F. Selleri, Phys. Lett. B 461, 123 (1999); Phys. Rev. A 61, 012106 (2000); F. Benatti and R. Floreanini, Phys. Rev. D 57, R1332 (1998); Eur. Phys. J. C 13, 267 (2000); R. A. Bertlmann, W. Grimus and B. C. Hiesmayr, Phys. Rev. D 60, 114032 (1999); Phys. Lett. A 289, 21 (2001); R. A. Bertlmann and B. C. Hiesmayr, Phys. Rev. A 63, 062112 (2001); Phys. Lett. A 332, 355 (2004); R. A. Bertlmann, K. Durstberger and B. C. Hiesmayr, Phys. Rev. A 68, 012111 (2003); A. Bramon and M. Nowakowski, Phys. Rev. Lett. 83, 1 (1999); B. Ancochea, A. Bramon and M. Nowakowski, Phys. Rev. D 60, 094008 (1999); N. Gisin and A. Go, Am. J. Phys. 69, 264 (2001); R. Dalitz and G. Garbarino, Nucl. Phys. B 606, 483 (2001).

[11] A. Bramon and G. Garbarino, Phys. Rev. Lett. 88, 040403 (2002).

[12] A. Bramon and G. Garbarino, Phys. Rev. Lett. 89, 160401 (2002).

[13] A. Afriat and F. Selleri, The Einstein, Podolsky and Rosen paradox in atomic, nuclear and particle physics (Plenum Press, New York, 1998).

[14] R. A. Bertlmann, Lect. Notes Phys. 689, 1 (2006) [quant-ph/0410028].

[15] A. Bramon, R. Escribano and G. Garbarino, Found. Phys. 36, 563 (2006) [quant-ph/0501069]; in Handbook on Neutral Kaon Interferometry at a $\phi$-factory, Frascati Physics Series (in press).

[16] The Second DaФne Physics Handbook edited by L. Maiani, G. Pancheri and N. Paver (INFN, Laboratori Nazionali di Frascati, Frascati, Italy, 1995).

[17] A. Angelopoulos et al., Phys. Rept. 374, 165 (2003).

[18] F. Ambrosino et al., Phys. Lett. B 642,315 (2006).

[19] A. Bramon, G. Garbarino and B. C. Hiesmayr, Phys. Rev. A 69, 062111 (2004).

[20] R. A. Bertlmann, A. Bramon, G. Garbarino and B. C. Hiesmayr, Phys. Lett. A 332, 355 (2004); A. Bramon, R. Escribano and G. Garbarino, J. Mod. Opt. 52, 1681 (2005).

[21] L. Hardy, Phys. Rev. Lett. 68, 2981 (1992); Phys. Rev. Lett. 71, 1665 (1993). 